\title{
Transurethral resection of bladder fumour (TURBT) as an optional treatment method on pheochromocytoma of the urinary bladder
}

\author{
Sun Gook Ahn, MD; ${ }^{*}$ Hoon Jang, MD; ${ }^{*}$ Dong Seok Han, MD;* Jung Uee Lee, MD; Seung Mo Yuk, MD* \\ *Departments of Hospital Urology, Daejeon St. Mary's Hospital, The Catholic University of Korea, Daejeon, Korea; 'Departments of Hospital Pathology, Daejeon St. Mary's Hospital, The Catholic University \\ of Korea, Daejeon, Korea
}

Cite as: Can Urol Assoc J 2013;7:E130-E134. http://dx.doi.org/10.5489/cuaj.255

\section{Abstract}

Pheochromocytoma of the urinary bladder is rare. We have experienced a case of unexpected pheochromocytoma of the urinary bladder in a 45-year-old female. An ultrasonographic, computed tomography scan and cystoscopic examination showed a submucosal bladder mass. After transurethral resection of bladder tumour was performed, the bladder mass was confirmed as pheochromocytoma by a pathologist. After surgery, the patient underwent a subsequent pelvic magnetic resonance imaging, positron emission tomography and I ${ }^{131}$-methyliodobenzylguanidine (I131-MIBG). An image study showed no residual tumour sites and no lymphatic metastasis. The patient has had no tumour recurrence and no voiding symptoms 3 years after the surgery.

\section{Introduction}

Pheochromocytoma arises from chromaffin cells and usually develops in the adrenal medulla. About $10 \%$ of pheochromocytomas can occur in ectopic or extra-adrenal sites ${ }^{1}$ along the sympathetic nervous system. Primary involvement of the urinary bladder is rare, accounting for less than $0.06 \%$ of all bladder tumours and less than $1 \%$ of all pheochromocytomas. ${ }^{2}$ Most pheochromocytomas (83\%) that invade the urinary bladder are hormonally active and lead to the characteristic symptoms of headache, palpitations, fainting, diaphoresis and hypertension after micturition due to increased catecholamine secretion during urination. Only $17 \%$ of pheochromocytomas of the urinary bladder are hormonally inactive. ${ }^{3}$ Patients with bladder pheochromocytoma can be diagnosed with confidence from a characteristic history of "micturitional attacks." We report a case of pheochromocytoma of the urinary bladder that failed to present with any typical symptoms presented and thus was not suspected before the operation.

\section{Case report}

A 45-year-old female was referred to the department of gynecology at our hospital from a private clinic with low abdominal pain and recurrent menorrhagia that had been present for the previous 4 months. The diagnosis of complex hyperplasia without atypia was made after endometrial curettage. An ultrasound study subsequently showed a $2.34 \times 2.40-\mathrm{cm}$ bladder mass and a $8.0 \times 7.0-\mathrm{cm}$ right ovarian cyst (Fig. 1a). The patient was transferred to the department of urology for proper management of the bladder mass. A computed tomography (CT) scan showed a $3.0 \times 2.4-\mathrm{cm}$ contrast-enhancing mass in the right anterior aspect of the bladder and an $8.0 \times 6.0-\mathrm{cm}$ right ovarian cystic mass (Fig. 1b). There was no history of hypertension and voiding symptoms. The physical examination showed unremarkable findings and the patient's blood pressure measured 130/80 mmHg. Routine laboratory tests were normal, and urine cytology for malignant cells was negative; only urinalysis showed microscopic hematuria. At a cystoscopic view, a submucosal mass was protruding from the right neck to the right lateral wall of the bladder, and no ulceration on the mucosa was observed. As the mass was small and welllocated at the submucosal layer, we performed transurethral resection of bladder tumour (TURBT) (Fig. 2a, Fig. 2b, Fig. 2c). Postoperative recovery was uneventful. Pathological diagnosis was unexpected. Microscopic examination of the resected bladder tumour showed that it was located in the submucosal layer and covered with normal urothelium; muscle invasion was not seen. The tumour cells were arranged in trabecules or nests outlined by capillaries and composed of large- to medium-sized, round to polygonal cells with abundant granular, eosinophilic cytoplasm and round to oval nuclei. The nucleus was centrally located and showed a mild pleomorphism with occasionally prominent nucleoli. A prominent vascular network was observed. Mitosis or necrosis were not seen (Fig. 3a, Fig. 3b).

Immunohistochemically, the tumour cells were positively stained with neuron-specific enolase (NSE), synaptophysin, 


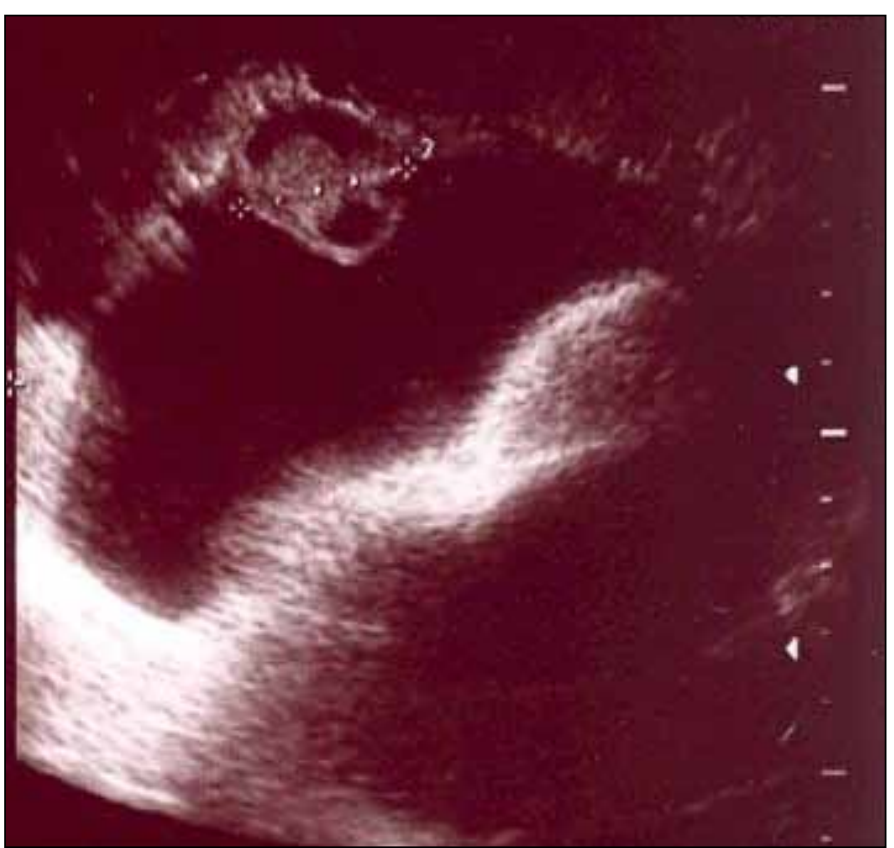

Fig. 1a. Ultrasonography revealed a $2.40 \times 2.34 \mathrm{~cm}$-sized urinary bladder mass.

S-100 protein, and CD56, although negatively stained with cytokeratin for carcinoma and periodic acid-Schiff (PAS) for granular cell tumour and chromogranin. The Ki-67 index was less than $1 \%$ (Fig. 4a, Fig. 4b, Fig. 4c, Fig. 4d). It was confirmed as extra-adrenal pheochromocytoma. After surgery, we checked positron emission tomography (PET), $\mathrm{I}^{131}$ MIBG and pelvic magnetic resonance imaging (MRI). There was no evidence of additional tumour sites, residual tumour or distant metastasis. Urinary and blood excretion of catecholamines and metanephrine was normal. Therefore, we did not perform further treatment, and no tumour recurrence has been found 3 years post-surgery.

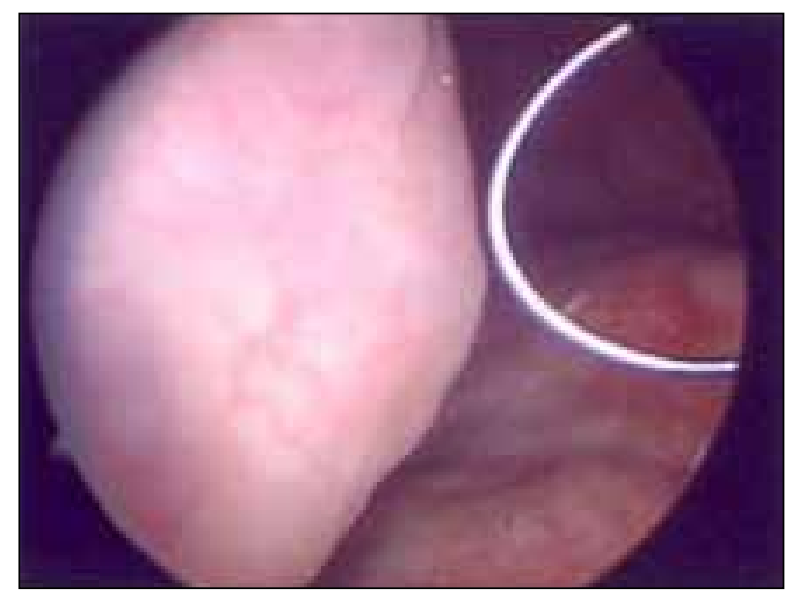

Fig. 2a. Cystoscopy showed a submucosal bladder tumour with protruding, non-papillary mass on normal mucosa.

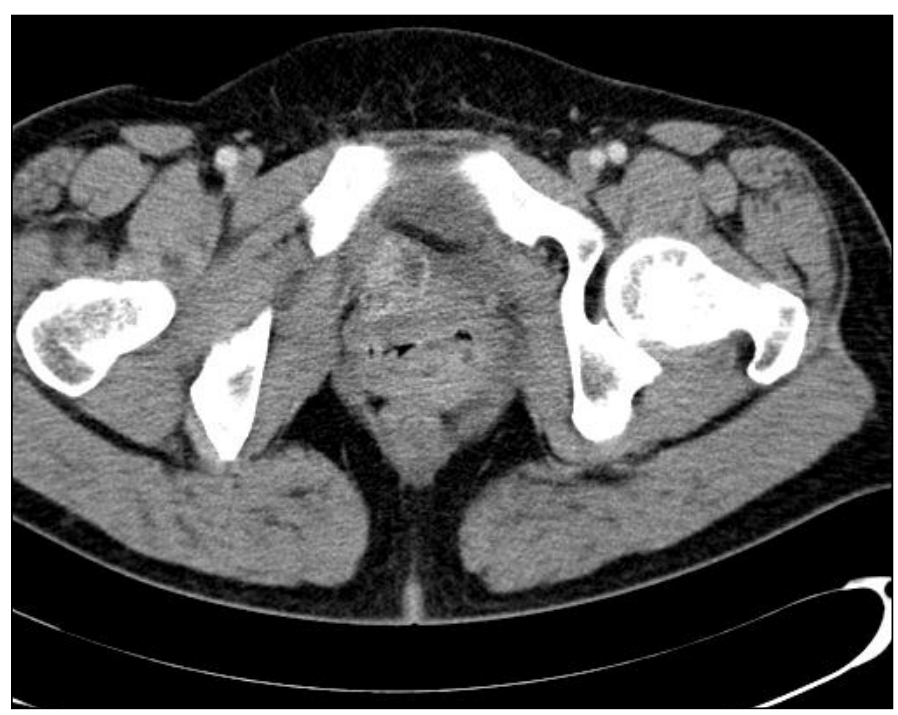

Fig. 1b. A computed tomography scan revealed a $3.0 \times 2.4 \mathrm{~cm}$-sized contrastenhancing mass in the right anterior aspect of the urinary bladder.

\section{Discussion}

Extra-adrenal pheochromocytoma of the urinary bladder is a rare neoplasm. They are histologically identical to pheochromocytomas at other sites. About $50 \%$ of patients have symptoms produced by the excessive secretion of catecholamines, sometimes associated with voiding. A characteristic "micturitional attack" consists of headache, palpitations, hypertension, blurred vision or sweating during voiding. ${ }^{3}$ These symptoms are due to increased catecholamine release in association with detrusor activity during micturition and are often the essential element required for the diagnosis of bladder pheochromocytoma. Bladder pheochromocytoma may not be suspected preoperatively if no typical symptoms are observed due to the rarity of the tumour.

The preoperative diagnosis of pheochromocytoma is supported by elevated levels of urine and blood catecholamines

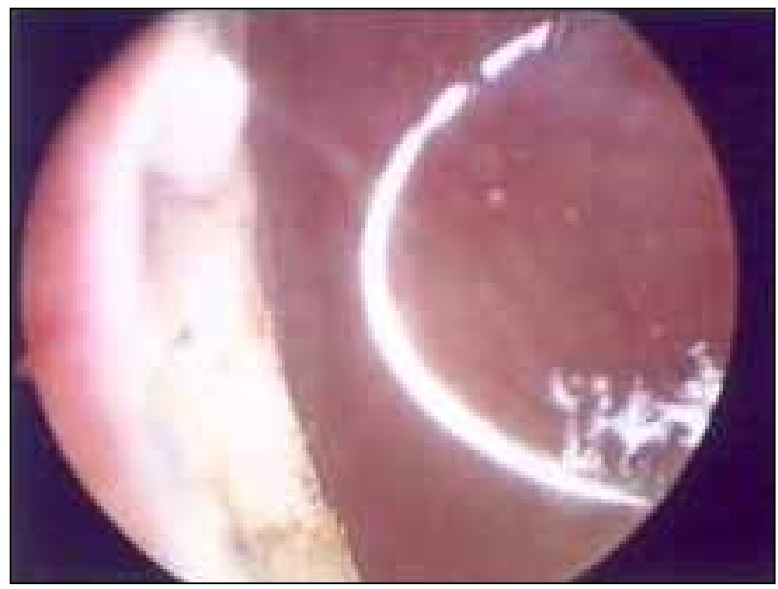

Fig. 2 b. During the transurethral resection of bladder pheochromocytoma. 
Ahn et al.

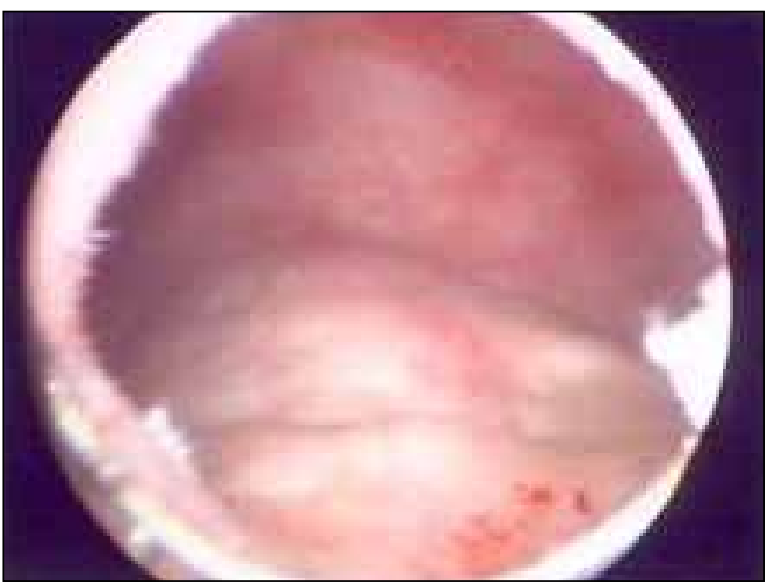

Fig. 2c. After transurethral resection of bladder pheochromocytoma.

and their metabolites, metanephrine and vanillyl mandelic acid (VMA). Urinary measurement of catecholamines and their metabolites has more diagnostic value than blood. ${ }^{4}$ Preoperative diagnosis and management are necessary to avoid catecholamine crises. To investigate for suspected case of pheochromocytoma, it is important to perform image analyses, such as $\mathrm{CT}$, for anatomical localization of the tumour and presence of lymphadenopathy. The functional scan, I'131-MIBG, is highly specific for the diagnostic confirmation of extra-adrenal pheochromocytoma and for the search for possible metastasis. ${ }^{4}$ In a recent study, PET instead of $\mathrm{I}^{131}$-MIBG was recommended for its sensitivity drops in extra-adrenal or malignant pheochromocytomas. ${ }^{5}$ Cystoscopy is another important way to identify bladder pheochromocytoma. Usually the tumour is located mainly in the submucosal layer protruding into the mucosa. The mucosal surface may be smooth but occasionally can be ulcerated.

When an unexpected pheochromocytoma manifests itself during even the simplest operations, operative mortality can be as high as $50 \% .{ }^{6}$ Therefore there is a need for early diagnosis before an operation, based on careful history-taking, paying careful attention to all reported symptoms, laboratory testing and image studies.

Microscopically, the cells are arranged in discrete nests, the "Zellballen" pattern, separated by a prominent vascular network. Cells are round with clear, amphophilic or acidophilic cytoplasm and ovoid nuclei. Mitoses are rare and usually absent. In about $10 \%$ of cases, small neuroblast-like cells are present, usually immediately beneath the normal urothelium. By immunohistochemistry, bladder pheochromocytomas react as they do at other sites, negative for epithelial markers and positive for the neuroendocrine markers, NSE, chromogranin, synaptophysin, and others. Flattened sustentacular cells can sometimes be highlighted in the periphery of the cell nests with S-100 protein. Ultrastructural features include dense core neurosecretory granules. ${ }^{7}$

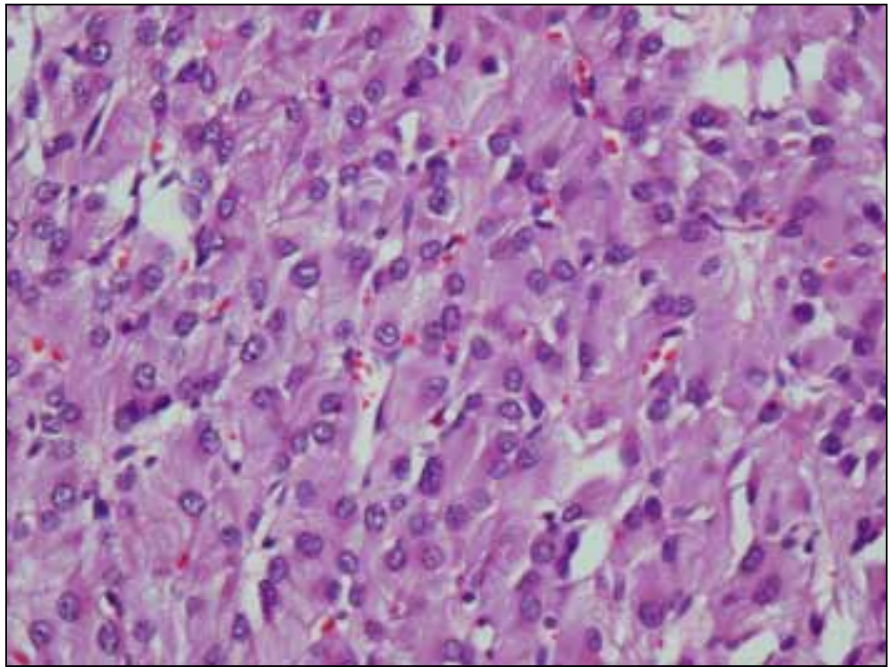

Fig. 3a. The cells are arranged in discrete nests, separated by a prominent vascular network (40x).

Currently, there are no reliable macroscopic or microscopic criteria to distinguish between benign and malignant tumours. ${ }^{8}$ However, the presence of necrosis, lymphovascular invasion, an increase in mitotic index, and DNA hyperdiploid or triploid mode and with loss of polyploidy may provide malignant potential.

The treatment of bladder pheochromocytoma is surgical; partial or radical cystectomy, which are known as the most effective management, is done in most cases of bladder pheochromocytoma. ${ }^{2,3}$ TURBT can irritate the tumour to induce the blood pressure to fluctuate; if resected incompletely, it often recurs. ${ }^{2}$ But in a review of some cases, bladder pheochromocytomas were removed by TURBT. ${ }^{3,9} \mathrm{We}$ also performed TURBT without any complications and follow-up showed no residual tumour and no recurrence 3 years post-

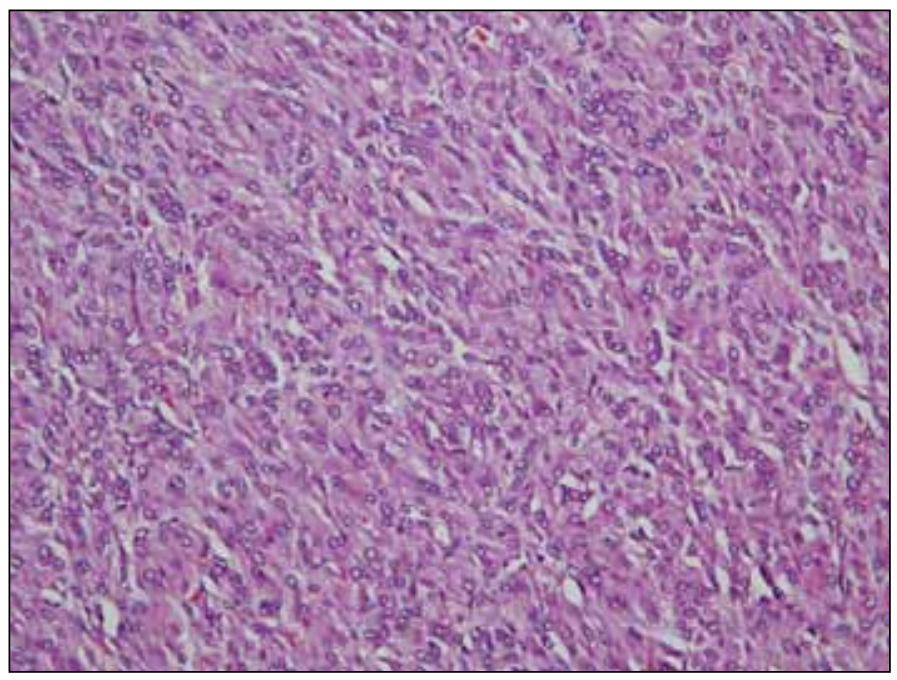

Fig. $3 \boldsymbol{b}$. Cells are large- to medium-sized, round to polygonal cells with abundant granular, eosinophilic cytoplasm and round to oval nuclei (200x). 
TURBT for pheochromocytoma of the urinary bladder

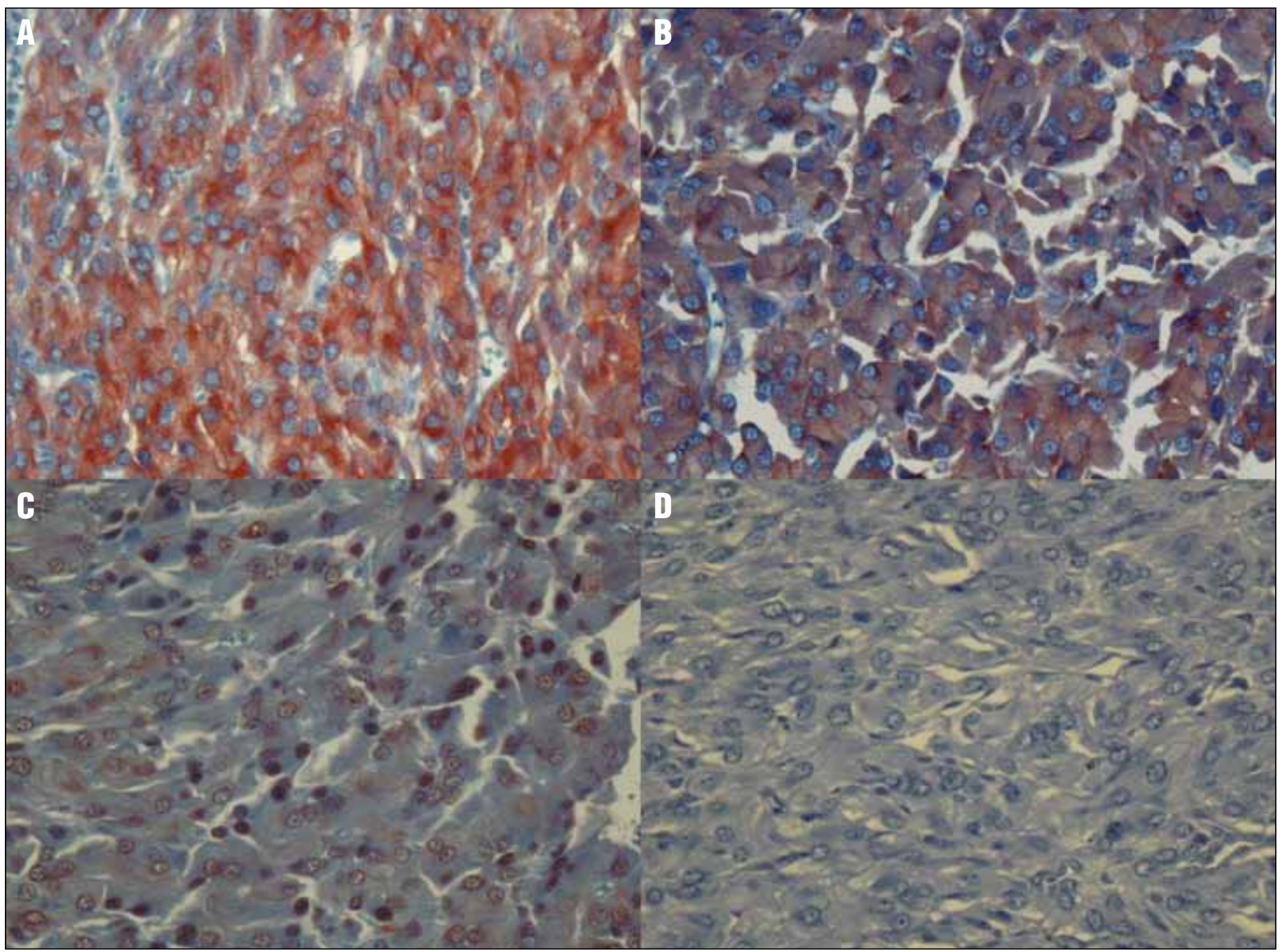

Fig. 4a. Immunohistochemical staining. (A) NSE, (B) Synaptophysin, (C) S-100 protein, (D) Cytokeratin.

operatively. In another study, patients with advanced stage tumours were at risk of recurrence, metastasis and death, but non-invasive tumours have had favourable outcomes after complete tumour removal. ${ }^{9}$

We believe that TURBT could be an viable minimally invasive procedure for pheochromocytoma of the bladder if the following conditions are present: (1) the tumour is small and non-invasive; (2) preoperative blood pressure is wellcontrolled and no present "micturition attacks" are occurring; and (3) an image study shows that the tumour is localized at the bladder without lymph node and distant metastasis. If the tumour is diagnosed as pheochromocytoma after the operation, 24-hour urinary fractionated catecholamines and metanephrines or fractionated plasma metanephrines should be measured. If these levels are normal, the biochemically active pheochromocytoma should be considered completely resected; elevated catecholamine and/or fractionated metanephrine levels should be considered indica- tive of remaining residual tumour or occult metastasis and requires additional treatment. ${ }^{10}$ The biochemically inactive tumour requires additional study for verification of residual tumour or metastatic lesions. An MRI should be considered for image analysis, as it is superior to a CT scan at finding submocosal tumour ${ }^{2}$ and as it is a reliable examination that can detect small size tumours. I $^{131}$-MIBG should be checked during a search for extra-adrenal pheochromocytomas and possible metastasis. In our case, we check MRI, I ${ }^{131}$-MIBG and PET; these showed no residual tumour, other tumour sites or metastasis.

Patients with bladder pheochromocytoma are recommended for long-term follow-up because of tumour recurrence. Patients should be measured 24-hour postoperatively for urinary catecholamines and metanephrines; in addition, a cystoscopy, CT and PET should be performed periodically. In our case, the patient was disease-free for 3 years according to urine chemistry and cystoscopy, CT and PET. 
Ahn et al.

\section{Conclusion}

TURBT can be an attractive option for bladder pheochromocytoma if the following conditions are present: (1) the mass is small with a well-located lesion, allowing for complete removal at TUR; (2) there is no evidence of metastasis and non-invasive, localized bladder at image study; and (3) the mass is hormonally inactive. After TURBT, image and metabolite studies should be performed to check for any residual tumour.

Competing interests: None declared.

This paper has been peer-reviewed.

\section{References}

1. Gifford RW, Manger WM, Bravo El. Pheochromocytoma. Endocrinol Metab Clin North Am 1994;23:387404.

2. Tsai C, Wu W, Chueh K, et al. Paraganglioma of the urinary bladder first presented by bladder bloody tamponade: two case reports and review of the literatures. Kaohsiung J Med Sci 2011;27:108-13. http://dx.doi.org/10.1016/i.kims.2010.05.005
3. Das S, Bulusu NV, Lowe P. Primary vesical pheochromocytoma. Urology 1983;21:20-5. http://dx.doi. org/10.1016/0090-4295(83)90116-4

4. Troncone L, Rufini V, Montemaggi P, et al. The diagnostic and therapeutic utility of radioiodinated metaiodobenzylguanidine(MIBG).5 years of experience. Eur J Nucl Med 1990;16:325-35. http:// dx.doi.org/10.1007/BF00842788

5. Havekes B, King K, Lai EW, et al. New imaging approaches to phaeochromocytomas and paragangliomas. Clin Endocrinol 2010;72:137-45. http://dx.doi.org/10.1111/i.1365-2265.2009.03648.x

6. Pullerits J, Ein S, Balfe JW. Anesthesia for phaeochromocytoma. Can J Anaesth 1988;35:526-34. http:// dx.doi.org/10.1007/BF03026905

7. Kato $H$, Suzuki $M$, Mukai $M$, et al. Clinicopathological study of pheochromocytoma of the urinary bladd er:immunohistochemical, flow cytometric and ultrastructural findings with review of the literature. Pathol Int 1999;49:1093-9. http://dx.doi.org/10.1046/j.1440-1827.1999.00985.x

8. Linnoila RI, Keiser HR, Steinberg SM, et al. Histopathology of benign versus malignant sympathoadrenal paragangliomas: clinicopathologic study of 120 cases including unusual histologic features. Hum Pathol 1990;21:1168-80. http://dx.doi.org/10.1016/0046-8177(90)90155-X

9. Baima C, Casetta G, Vella R, et al. Bladder pheochromocytoma: A 3-year follow-up after transurethral resection(TURB). Urol Int 2000;65:176-8. http://dx.doi.org/10.1159/000064868

10. Young WF. Paragangliomas: Clinical overview. Ann NY Acad Sci 2006;1073:21-9. http://dx.doi. org/10.1196/annals.1353.002

Correspondence: Dr. Seung Mo Yuk, Department of Hospital Urology, Dae-Jeon St. Mary's Hospital, The Catholic University of Korea, 520-2 Daeheung-dong, Jung-ku, Dae-Jeon, Korea, 301-723; fax: 82-042-220-9051; doctor6@hanmail.net 